\title{
COMPUTATIONAL STUDY OF NONLINEAR OPTICAL PROPERTIES OF BENZOXAZOLES EXHIBITING EXCITED STATE PROTON TRANSFER
}

\author{
Y. Syetov \\ Oles Honchar Dnipro National University, Dnipro, Ukraine \\ e-mail:setov2003@yahoo.com
}

Hyperpolarizability of molecules of 2-(2'-hydroxyphenyl)benzoxazole and 2,5-bis(2benzoxazolyl)phenol is calculated by density functional theory quadratic response method for static electric field and hyper-Rayleigh scattering at $532 \mathrm{~nm}$. It is found that 2,5-bis(2benzoxazolyl)phenol demonstrates the hyperpolarizability about ten times larger than 2-(2'hydroxyphenyl)benzoxazole. Dominant component of the hyperpolarizability tensor corresponds to the long axis of the molecule.

Keywords: nonlinear optics, excited state proton transfer, benzoxazoles, density functional theory.

Received 08.09.2019; Received in revised form 11.10.2019; Accepted 15.10.2019

\section{Introduction}

Organic substances are considered as possible nonlinear optical materials due to large values of their nonlinear susceptibilities caused by weak coupling of $\pi$ electrons with nuclei in the molecules [1]. In dye-doped polymers and molecular crystals, where the interaction between the molecular units is significantly weaker than intramolecular forces, the macroscopic nonlinear optical susceptibility can be estimated as a sum over microscopic hyperpolarizabilities of the molecules transformed into the corresponding coordinate system. To obtain macroscopic non-centrosymmetric material, the molecules with a large permanent dipole moment can be oriented properly in the polymer matrix by strong electric field [2]. The alignment of the dye molecules is observed upon incorporation into carbon nanotubes [3]. Quadratic nonlinear response of the molecules to the electric field of the light wave can be observed in solution as hyper-Rayleigh scattering [2].

Organic compounds 2-(2'-hydroxyphenyl)benzoxazole (HBO) and 2,5-bis(2benzoxazolyl)phenol (DBP) (Fig. 1) are benzoxazoles with intramolecular hydrogen bonds undergoing photoinduced excited state intramolecular proton transfer (ESIPT). The enol structures with the $\mathrm{OH} \ldots \mathrm{N}$ hydrogen bonds shown in Fig. 1 have the lowest energy in the ground state. The molecular structure of $\mathrm{HBO}$ and DBP is non-centrosymmetric so that they are expected to demonstrate second-order nonlinear optical properties. Nonlinear optical behavior of the compound $\mathrm{HBO}$ in solutions has been found as hyper-Rayleigh scattering (quadratic nonlinearity) [4] and nonlinear refractive index (cubic nonlinearity) [5].

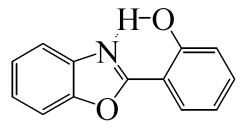

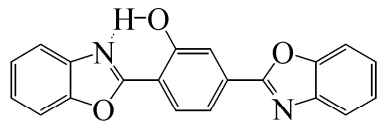

b

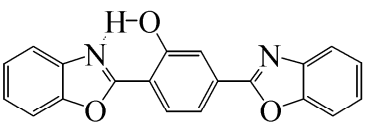

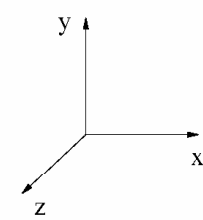

Fig. 1. Molecular structure of HBO (a), two conformations of DBP (b, c) and coordinate system (d) used for calculations. 


\section{Calculations details}

Induced dipole moment of the molecule can be expressed as a power series of the electric field $E$ [2]:

$$
\mu_{i}=\alpha_{i k} E_{k}+\beta_{i j k} E_{j} E_{k}+\gamma_{i j k l} E_{j} E_{k} E_{l}+\ldots,
$$

where $\alpha_{i k}$ is polarizability, $\beta_{i j k}$ is first hyperpolarizability, $\gamma_{i j k l}$ is second hyperpolarizability; repeated indices are summed over Cartesian coordinates $x, y, z$.

Induced second-order molecular dipole moment with the oscillation frequency $\omega$ is defined as

$$
\mu_{i}(\omega)=\beta_{i j k}\left(-\omega ; \omega_{1}, \omega_{2}\right) E_{j}\left(\omega_{1}\right) E_{k}\left(\omega_{2}\right)
$$

where $\omega= \pm \omega_{1} \pm \omega_{2}$.

In this study we calculate static hyperpolarizability $\beta_{i j k}(0 ; 0,0)$ and dynamic hyperpolarizability $\beta_{i j k}(-2 \omega ; \omega, \omega)$ that corresponds to the induced dipole moment $\mu_{i}(2 \omega)$. The total hyperpolarizability of the molecule is calculated by the relation

$$
\beta_{\text {total }}=\left(\beta_{x}^{2}+\beta_{y}^{2}+\beta_{z}^{2}\right)^{1 / 2}
$$

where $\beta_{i}=\beta_{i i i}+\frac{1}{3} \sum_{i \neq j}\left(\beta_{i j j}+\beta_{j i j}+\beta_{j j i}\right)[6]$.

When the permutation Kleinmann symmetry is hold, the relation is expressed as

$$
\beta_{\text {total }}=\left[\left(\beta_{x x x}+\beta_{x y y}+\beta_{x z z}\right)^{2}+\left(\beta_{y y y}+\beta_{y z z}+\beta_{y x x}\right)^{2}+\left(\beta_{z z z}+\beta_{z y y}+\beta_{z x x}\right)^{2}\right]^{1 / 2}
$$

The frequency $\omega$ of the pumping wave for dynamic hyperpolarizability calculations is chosen to be $1.17 \mathrm{eV}(1064 \mathrm{~nm})$, the frequency of emission of YAG: $\mathrm{Nd}^{+}$laser used in [4]. All components of $\beta_{i j k}$ are presented in the coordinate system of the principal axes of the inertia moment tensors. Approximate directions of the coordinate axes are shown in Fig. 1d. The principal axis $O x$ deviate from the bond linking H-bonded benzoxazole and phenol fragments by about $5^{\circ}$ in the case of $\mathrm{HBO}$, about $2^{\circ}$ for structure $\mathrm{b}$ of DBP, and almost parallel for structure $\mathrm{c}$ of DBP.

All calculations are performed with the DALTON program [7, 8]. Components of the hyperpolarizability tensors are obtained by quadratic response density functional theory method implemented in DALTON using the Becke three-parameter Lee-YangParr (B3LYP) functional [9] and 6-31G(d, p) basis set.

\section{Results and discussion}

Calculated components of the hyperpolarizability tensor $\beta_{i j k}$ are collected in Table 1. Since the molecules of $\mathrm{HBO}$ and DBP are planar (posses $\mathrm{C}_{\mathrm{s}}$ symmetry), the components of $\beta_{i j k}$ with odd number of indices $z$ (the axis $\mathrm{O} z$ is perpendicular to the molecular plane) are equal to zero. Static hyperpolarizabilities strictly obey Kleinman symmetry in relation to permutation of all indices $i, j, k$, whereas dynamic hyperpolarizabilities demonstrate deviation from the Kleinman symmetry due to dispersion. The deviation is more pronounced for the compound DBP (see Table 1). In the case of DBP, the frequency of the second harmonic $2.34 \mathrm{eV}$ is closer to the resonance 
(absorption transition) than for HBO. The first absorption transitions are calculated to be at $3.92 \mathrm{eV}$ for $\mathrm{HBO}$ and $3.44 \mathrm{eV}$ for DBP. For the molecule of $\mathrm{HBO}$ the components $\beta_{x x y}$ and $\beta_{y x x}$ demonstrate stronger dispersion than $\beta_{x x x}$ and $\beta_{y y y}$. The presence of the second benzoxazole fragment in the molecule of DBP substantially increases the value $\beta_{x x x}$ in relation to $\mathrm{HBO}$ and $\beta_{x x x}$ of DBP is more than 10 times larger than other components of the tensor. The molecule of DBP has two possible structures which are formed by the rotation of the non-hydrogen-bonded moiety and are close in energy (Fig. $1 \mathrm{~b}$ and $\mathrm{c}$ ). The less stable structure $\mathrm{c}$ demonstrates larger components $\beta$ related to the short axes $y$ than the structure $\mathrm{b}$, whereas the values of the component $\beta_{x x x}$ are close.

The molecules of HBO and DBP possess dipole moments of 2.13 and $1.67 \mathrm{D}$ correspondingly. The value for the structure $\mathrm{c}$ of DBP is $3.18 \mathrm{D}$. The directions of the dipole moment are not parallel to the long axes of the molecules; the angles are $52^{\circ}$ (HBO), $23^{\circ}$ (DBP, structure b) and $61^{\circ}$ (DBP, structure c).

Table 1

Calculated components of hyperpolarizability tensor HBO, DBP and $p$-nitroaniline (PNA) (the values are presented in atomic units, 1 a.u. $=3.206 \times 10^{-53} \mathrm{C}^{3} \mathrm{~m}^{3} \mathrm{~J}^{-2}$ )

\begin{tabular}{|c|c|c|c|c|c|c|}
\hline Compound & Component & $\begin{array}{c}\text { Static } \beta, \\
\text { a.u. }\end{array}$ & $\begin{array}{c}\text { Dynamic } \beta, \\
\text { a.u. }\end{array}$ & Component & $\begin{array}{c}\text { Static } \beta, \\
\text { a.u. }\end{array}$ & $\begin{array}{c}\text { Dynamic } \beta, \\
\text { a.u. }\end{array}$ \\
\hline \multirow{4}{*}{ HBO } & yyy & 100 & 113 & xxx & 220 & 239 \\
\cline { 2 - 7 } & xyy & -29 & -8 & zyz zzy & 2 & 2 \\
\cline { 2 - 7 } & yyx yxy & -29 & -4 & zxz zzx & -3 & -4 \\
\cline { 2 - 7 } & xyx xxy & 68 & 175 & xzz & -3 & -5 \\
\cline { 2 - 7 } & yxx & 68 & 163 & total & 273 & 335 \\
\hline \multirow{4}{*}{ DBP } & yyy & 89 & 116 & xxx & 2453 & 5404 \\
\cline { 2 - 7 } & xyy & -77 & -39 & zyz zzy & 0 & 0 \\
\cline { 2 - 7 } & yyx yxy & -77 & -68 & zxz zzx & 1 & 2 \\
\cline { 2 - 7 } & xyx xxy & -150 & -363 & xzz & 1 & 2 \\
\cline { 2 - 7 } & yxx & -150 & -460 & total & 2377 & 5355 \\
\cline { 2 - 7 } & yyy & 109 & 142 & xxx & 2455 & 5554 \\
\cline { 2 - 7 } & xyy & -89 & -65 & zzy zzy & 4 & 4 \\
\cline { 2 - 7 } & yyx yxy & -89 & -91 & zxz zzx & 1 & 2 \\
\cline { 2 - 7 } & xyx xxy & -350 & -868 & xzz & 1 & 1 \\
\cline { 2 - 7 } & yxx & -350 & -811 & total & 2379 & 5518 \\
\hline \multirow{4}{*}{ structure d } & xyy & -145 & -209 & xxx & 1506 & 2521 \\
\cline { 2 - 7 } & yyx yxy & -145 & -161 & zxz zzx & -3 & -3 \\
\cline { 2 - 7 } & xzz & -3 & -4 & total & 1360 & 2341 \\
\hline
\end{tabular}

The hyperpolarizabilities of HBO and DBP are compared with the hyperpolarizability of $p$-nitroaniline (PNA) which is a reference compound frequently used in computational and experimental study on nonlinear optical properties of organic substances. The value of total polarizability of HBO is substantially lower than PNA, the ratio is 0.2 (static) and 0.14 (dynamic). The predicted total hyperpolarizability of a molecule of DBP is two times more than the hyperpolarizability of PNA. HBO hyperpolarizability estimation in the work [4] from the intensity of hyper-Rayleigh scattering gives the value 3 times larger than hyperpolarizability of PNA. However, the measurements were performed in acetone where the interaction with polar solvent molecules could influence the hyperpolarizability. General solvent effects are additional electric field caused by solvent polarization by the solvated molecules and shift of the energy of absorption transitions [10]. In the case of HBO, polar solvents affect structure of the molecules stabilizing structures with an $\mathrm{OH} . . . \mathrm{O}$ hydrogen bonds and forming complexes with intermolecular hydrogen bonds [11]. 


\section{Conclusions}

Molecular hyperpolarizability tensors are calculated by quadratic response density functional theory for 2-(2'-hydroxyphenyl)benzoxazole and 2,5-bis(2benzoxazolyl)phenol. The presence of the second beznoxazole fragment in the molecule of 2,5-bis(2-benzoxazolyl)phenol substantially increase the value of polarizability making it relevant for nonlinear optical applications. The dominant component of the polarizability tensor is found for the electric field directed parallel to the long axis of the molecule. For prediction of nonlinear properties of the molecules in the condensed state local field effect and interaction with the surrounding molecules should be considered.

\section{References}

1. Kajzar, F. Organic Nonlinear Optics: Historical Survey and Current Trends / F. Kajzar, J. Zyss // Nonlinear Optics and Quantum Optics. - 2012. - Vol. 43. - P. 31 - 95.

2. Kuzyk, M. G. Theory of Molecular Nonlinear Optics / M.G. Kuzyk, K.D. Singer, G.I. Stegeman // Advances in Optics and Photonics. - 2013. - Vol. 5. - P. 4 - 82.

3. Zhang, Y. Dipoles align inside a nanotube / Y. Zhang, W.J. Blau // Nature Nanotechnology. - 2015. - Vol. 10. - P. 205 - 206.

4. Hillebrand, S. First hyperpolarizability in proton-transfer benzoxazoles: computer-aided design, synthesis and study of a new model compound / S. Hillebrand, M. Segala, T. Buckup, R.R.B. Correia, F. Horowitz, V. Stefani // Chem. Phys. - 2001. - Vol. 273. - P. 1 - 10.

5. Zhang, G. Optical switching of 2-(2'-hydroxyphenyl) benzoxazole in different solvents / G. Zhang, H. Wang, Y. Yu, F. Xiong, G. Tang, W. Chen // Appl. Phys. B. - 2003. - Vol. 76. P. $677-681$.

6. Stähelin, M. A comparison of calculated and experimental hyperpolarizabilities for acetonitrile in gas and liquid phases / M. Stähelin, C.R. Moylan, D.M. Burland, A. Willetts, J.E. Rice, D.P. Shelton, E.A. Donley // J. Chem. Phys. - 1993. - Vol. 98. - P. 5595 - 5603.

7. Aidas, K. The Dalton quantum chemistry program / K. Aidas, C. Angeli, K.L. Bak, V. Bakken, R. Bast, L. Boman, O. Christiansen, R. Cimiraglia, S. Coriani, P. Dahle, E.K. Dalskov, U. Ekstroem, T. Enevoldsen, J. J. Eriksen, P. Ettenhuber, B. Fernandez, L. Ferrighi, H. Fliegl, L. Frediani, K. Hald, A. Halkier, C. Haettig, H. Heiberg, T. Helgaker, A.C. Hennum, H. Hettema, E. Hjertenaes, S. Hoest, I.-M. Hoeyvik, M.F. Iozzi, B. Jansik, H.J. Aa. Jensen, D. Jonsson, P. Joergensen, J. Kauczor, S. Kirpekar, T. Kjaergaard, W. Klopper, S. Knecht, R. Kobayashi, H. Koch, J. Kongsted, A. Krapp, K. Kristensen, A. Ligabue, O.B. Lutnaes, J.I. Melo, K.V. Mikkelsen, R.H. Myhre, C. Neiss, C.B. Nielsen, P. Norman, J. Olsen, J.M.H. Olsen, A. Osted, M.J. Packer, F. Pawlowski, T.B. Pedersen, P.F. Provasi, S. Reine, Z. Rinkevicius, T.A. Ruden, K. Ruud, V. Rybkin, P. Salek, C.C.M. Samson, A. Sanchez de Meras, T. Saue, S.P.A. Sauer, B. Schimmelpfennig, K. Sneskov, A.H. Steindal, K.O. Sylvester-Hvid, P.R. Taylor, A.M. Teale, E.I. Tellgren, D.P. Tew, A.J. Thorvaldsen, L. Thoegersen, O. Vahtras, M.A. Watson, D.J.D. Wilson, M. Ziolkowski, H. Agren // WIREs Comput. Mol. Sci. - 2014. - Vol. 4. - P. 269 284.

8. Dalton, a Molecular Electronic Structure Program. Release Dalton2017.alpha (2017), http://daltonprogram.org.

9. Becke, A.D. Density-functional thermochemistry. III. The role of exact exchange / A.D. Becke // J. Chem. Phys. - 1993.- Vol. 98. - P. 5648 - 5652.

10. Woodford, J.N. Solvent Dependence of the First Molecular Hyperpolarizability of pNitroaniline Revisited / J.N. Woodford, M.A. Pauley, C.H. Wang // J. Phys. Chem. A. - 1997. Vol. 101. - P. $1990-1992$.

11. Wang, H. Femtosecond fluorescence upconversion studies of excited-state protontransfer dynamics in 2-(2'-hydroxyphenyl)benzoxazole (HBO) in liquid solution and DNA / H. Wang, H. Zhang, O.K. Abou-Zied, C. Yu, F.E. Romesberg, M. Glasbeek // Chem. Phys. Lett. 2003. - Vol. 367. - P. 599 - 608. 